\title{
Atividade microbiana em solos sob doses de lodo de estação de tratamento de efluentes de um aterro industrial
}

\author{
Soil microbial activity under wastewater treatment \\ plant sludge doses from an industrial landfill
}

\author{
Leonardo Capeleto de Andrade ${ }^{I}$ Robson Andreazza ${ }^{I}$ \\ Flávio Anastácio de Oliveira Camargo ${ }^{I}$
}

\section{RESUMO}

A destinação final adequada dos resíduos sólidos industriais é um dos principais problemas ambientais. A disposição de resíduos orgânicos em solos pode ser recomendada, dado o potencial corretivo e fertilizante deste. Assim, o objetivo deste estudo foi avaliar o efeito em solos da adição de doses de lodo de estação de tratamento de efluentes (ETE) de um aterro industrial na atividade microbiana, avaliada pela mineralização de carbono. Foi desenvolvido experimento em frascos respirométricos, analisando a mineralização de carbono em três solos, com cinco doses de lodo durante 91 dias de incubação. A aplicação de lodo com $2 \mathrm{Mg} \mathrm{ha} \mathrm{g}^{-1}$ causou menores impactos à microbiota do solo, não apresentando diferenças em relação ao tratamento controle. Os valores de carbono mineralizado aumentaram com o tempo de incubação em função da adição do lodo, proporcionalmente ao aumento das doses de lodo. A atividade microbiana aumentou com a aplicação de doses de lodo de ETE de aterro industrial, sendo a mineralização de carbono proporcional às doses adicionadas.

Palavras-chave: biodegradação, tratamento de resíduos, mineralização de carbono, lodos de ETE.

\section{ABSTRACT}

The final disposal of industrial solid waste is a major environmental problem. Disposal of organic wastes in soils can be recommended as corrective and fertilizer. The objective of this study was to evaluate the effect of different doses of wastewater treatment plant sludge (WWTP) from an industrial landfill on soils based in microbial activity measured by carbon mineralization. The experiment was developed in respirometric flasks, analyzing carbon mineralization in three soils with five doses of sludge during 91 days of incubation. Application of sewage sludge with $2 \mathrm{Mg} \mathrm{ha}^{-1}$ caused minor impacts on soil microorganisms, without statistical differences to the control treatment. Amounts of mineralized carbon increased with the time course due to the addition of sludge, proportional to the doses of sludge. Microbial activity increased with the application of WWTP sludge doses of industrial landfill, with mineralization proportional to the increase in carbon added doses.

Key words: biodegradation, waste treatment, carbon mineralization, WWTP sludge.

\section{INTRODUÇÃO}

A destinação final de resíduos sólidos industriais é um dos principais problemas ambientais, devido ao seu potencial de contaminação. Com a evolução das políticas e da ética ambiental, a destinação final adequada dos resíduos é estimulada, buscando-se assim novas tecnologias e destinos para suprir a demanda de resíduos gerados. Uma das soluções para este passivo ambiental pode ser a aplicação desses resíduos no solo.

A disposição de resíduos orgânicos em solos pode ser recomendada, dado os potenciais corretivos e fertilizantes desses materiais, propiciando a redução na aplicação de insumos minerais e melhoria da qualidade do solo, visto que os resíduos orgânicos podem atuar como condicionadores de solo (FERREIRA et al., 2003; SILVA, 2009). Esta forma de disposição final atende aos pressupostos da "disposição final ambientalmente adequada”, preceito da Política Nacional de Resíduos Sólidos (BRASIL, 2010).

Efeitos positivos e negativos, na aplicação de lodos em solos, podem ser observados,

\footnotetext{
'Universidade Federal do Rio Grande do Sul (UFRGS), Porto Alegre, RS, Brasil

IIUniversidade Federal de Pelotas (UFPel), 96010-280, Pelotas, RS, Brasil. E-mail: robsonandreazza@yahoo.com.br. Autor para correspondência.
} 
dependendo das doses utilizadas. Efeitos positivos no desenvolvimento de plantas e atividade microbiana já foram apresentados por vários autores (SELBACH et al., 1991; CAVALLET \& SELBACH, 2008; GIANELLO et al., 2011; SEGATTO et al., 2012). Em estudos de SELBACH et al. (1991), PASSIANOTO et al. (2001), FERREIRA et al. (2003) e CAVALLET \& SELBACH (2008), a adição de lodos de curtume em solos estimulou a atividade microbiana e não influenciou na população de microrganismos. Outro resíduo orgânico, como o de indústria fumageira, pode promover a atividade microbiana, além de melhorar as condições de $\mathrm{pH}$ e mineralização de nitrogênio no solo (LAUSCHNER et al., 2013). Por outro lado, a adição de lodos e ou resíduos orgânicos contendo excesso de metais pesados, pode diminuir a atividade microbiana do solo pela sua toxidez e também afetar outros organismos, como plantas (SEGATTO et al., 2012) e até mesmo animais. Em relação aos lodos de Estação de Tratamento de Efluentes (ETE) de aterros industriais, suas características dependerão dos resíduos dispostos nas células.

A aplicação em solo de lodo de ETE de aterro industrial pode ter efeitos positivos sobre a atividade microbiana. Assim, o objetivo deste estudo foi avaliar o efeito da adição de doses de lodo de ETE de um aterro industrial em solos na atividade microbiana, avaliada pela mineralização de carbono.

\section{MATERIAL E MÉTODOS}

Nos ensaios, foram utilizados três solos: Pvd* - Argissolo Vermelho Distrófico, impactado; Pvd - Argissolo Vermelho Distrófico (Unidade de Mapeamento São Jerônimo), não impactado; e Pvad - Argissolo Vermelho-Amarelo Distrófico espessarênico abrúptico (Unidade de Mapeamento Itapuã) (STRECK et al., 2008; EMBRAPA, 2009). $\mathrm{O}$ solo $\mathrm{Pvd}^{*}$ foi coletado em uma área de extração de argila (2940'36.7'’ $\left.51^{\circ} 12^{\prime} 45.3^{\prime \prime} \mathrm{W}\right)$ utilizada na compactação dos leitos das células do aterro, sendo predominantemente derivado dos horizontes A e C, com poucos vestígios do horizonte B. O solo Pvd foi coletado na Estação Experimental Agronômica (EEA/UFRGS), em Eldorado do Sul (RS), em área de campo nativo (3005'32.6”S 5140’32.9'W). O solo Pvad foi coletado em Itapuã, distrito de Viamão (RS), nas proximidades da Estrada Coronel Acrísio Martins Prates (30¹0'48.0”S 5059'47.2”W). Foi coletada a camada de $0-20 \mathrm{~cm}$ dos solos, sendo caracterizados conforme tabela 1. As caracterizações físico-químicas dos solos foram realizadas no Laboratório de Análises do Solo, da Faculdade de Agronomia, UFRGS,
Tabela 1 - Caracterização dos solos Pvd*, Pvd e Pvad.

\begin{tabular}{|c|c|c|c|c|}
\hline Atributos & Unidade & Pvd* & Pvd & Pvad \\
\hline Argila & $\%$ & 13 & 26 & 12 \\
\hline $\mathrm{pH} \mathrm{H} \mathrm{H}_{2} \mathrm{O}(1: 1)$ & - & 6,9 & 5,4 & 5,2 \\
\hline SMP & - & 7,1 & 6,1 & 6,5 \\
\hline M.O. & $\%$ & 1,9 & 1,9 & 1,3 \\
\hline $\mathrm{Al}_{\text {troc }}$ & cmolc dm ${ }^{-3}$ & 0 & 6,4 & 1,2 \\
\hline $\mathrm{Ca}_{\text {troc }}$ & cmolc $\mathrm{dm}^{-3}$ & 0,2 & 2,3 & 1,0 \\
\hline $\mathrm{Mg}_{\text {troc }}$ & cmolc dm ${ }^{-3}$ & 0,6 & 0,6 & 0,3 \\
\hline $\mathrm{Al}+\mathrm{H}$ & cmolc $\mathrm{dm}^{-3}$ & 1,2 & 3,9 & 2,5 \\
\hline СТC & cmolc $\mathrm{dm}^{-3}$ & 8,9 & 7,5 & 3,4 \\
\hline Bases & $\%$ Sat. CTC & 86 & 47 & 26 \\
\hline $\mathrm{Al}$ & $\%$ Sat. CTC & 0,0 & 5,3 & 39,7 \\
\hline $\mathrm{Ca} / \mathrm{Mg}$ & - & 5 & 2,3 & 1,7 \\
\hline $\mathrm{Ca} / \mathrm{K}$ & - & 86 & 11 & 7 \\
\hline $\mathrm{Mg} / \mathrm{K}$ & - & 16 & 4,9 & 4,2 \\
\hline $\mathrm{P}$ & $\mathrm{mg} \mathrm{dm} \mathrm{m}^{-3}$ & 10 & 5,5 & 2,9 \\
\hline K & $\mathrm{mg} \mathrm{dm} \mathrm{m}^{-3}$ & 29 & 80 & 28 \\
\hline S & $\mathrm{mg} \mathrm{dm} \mathrm{d}^{-3}$ & 12 & 7,4 & 6,2 \\
\hline $\mathrm{Zn}$ & $\mathrm{mg} \mathrm{dm} \mathrm{m}^{-3}$ & 12 & 7,7 & 3,9 \\
\hline $\mathrm{Cu}$ & $\mathrm{mg} \mathrm{dm} \mathrm{d}^{-3}$ & 1,8 & 1,1 & 0,5 \\
\hline $\mathrm{Mn}$ & $\mathrm{mg} \mathrm{dm} \mathrm{m}^{-3}$ & 12 & 35 & 21 \\
\hline $\mathrm{Na}$ & $\mathrm{mg} \mathrm{dm^{-3 }}$ & 8 & 5 & 6 \\
\hline
\end{tabular}

Pvd* (impactado); Pvd - UM São Jerônimo; Pvad - UM Itapuã.

segundo metodologia descrita por TEDESCO et al. (1995). O lodo utilizado neste estudo foi coletado nos leitos de secagem da Estação de Tratamento de Efluentes (ETE) de um aterro industrial, localizado no município de Estância Velha, região metropolitana de Porto Alegre (RS), em área de grande concentração de empresas do ramo coureiro-calçadista. O material foi seco em estufa a $65^{\circ} \mathrm{C} \pm 4^{\circ} \mathrm{C}$, moído, tamisado ( $2 \mathrm{~mm})$ e caracterizado.

A caracterização do Lodo demonstrou as seguintes características físico-químicas: Carbono orgânico (7,3\%); Nitrogênio (3,9\%), Fósforo total $(0,19 \%)$; Potássio total $(0,31 \%)$; Cálcio total $(20 \%)$; Magnésio total $(0,81 \%)$; Enxofre total $(1,2 \%)$; Ferro total (4,7\%); Sódio total $(2,6 \%)$; Cobre total $(23 \mathrm{mg}$ $\left.\mathrm{kg}^{-1}\right)$; Zinco total $\left(32 \mathrm{mg} \mathrm{kg}^{-1}\right)$; Manganês total $(658 \mathrm{mg}$ $\left.\mathrm{kg}^{-1}\right)$; Cádmio total $\left(0,3 \mathrm{mg} \mathrm{kg}^{-1}\right)$; Cromo trivalente $\left(602 \mathrm{mg} \mathrm{kg}^{-1}\right)$; Cromo hexavalente $\left(<1 \mathrm{mg} \mathrm{kg}^{-1}\right)$; Níquel total $\left(38 \mathrm{mg} \mathrm{kg}^{-1}\right)$; Arsênio total $\left(12 \mathrm{mg} \mathrm{kg}^{-1}\right)$; Mercúrio $\left(0,01 \mathrm{mg} \mathrm{kg}^{-1}\right)$; Poder de neutralização (49\%); $\mathrm{pH} \mathrm{H}_{2} \mathrm{O}$ $(1: 1)(7,9)$; Condutividade elétrica $\left(12,8 \mathrm{dS} \mathrm{m}^{-1}\right)$.

O ensaio de Mineralização de Carbono visou à avaliação e quantificação da atividade microbiana e o monitoramento da mineralização de carbono, ao longo do tempo e em diferentes doses de lodo, utilizando-se, para isso, a metodologia 
proposta por ANDERSON \& DOMSCH (1978). $\mathrm{O}$ método respirométrico é uma forma indireta de avaliação da atividade microbiana (CETESB, 1990) e da decomposição dos resíduos orgânicos no solo (ABNT, 1999) e tem sido utilizada para avaliar os efeitos de metais pesados sobre a microbiota do solo. Foram determinados também a Biomassa microbiana (BMS-C), a Respiração Basal do Solo (BMS-5) e o Quociente Metabólico $\left(q \mathrm{CO}_{2}\right)$. Para determinação da BMS-C, utilizou-se metodologia (i/i) proposta por FERREIRA et al. (1999). Para determinação do $q \mathrm{CO}_{2}$, utilizou-se equação proposta por SILVA et al. (2009). O experimento foi conduzido utilizando-se delineamento inteiramente casualizado, com três solos (Pvd*, Pvd e Pvad), cinco doses de lodo (0, 2, 10, 25, e $\left.50 \mathrm{Mg} \mathrm{ha}^{-1}\right)$ e três repetições. Os solos selecionados são encontrados na região de geração do resíduo estudado, tendo variações em suas características. As doses utilizadas foram baseadas em estudos prévios com o resíduo. Foram utilizados frascos respirométricos de vidro $(1,1 \mathrm{~L})$, hermeticamente fechados, com $200 \mathrm{~g}$ de solo (massa úmida), com a umidade dos solos ajustada a $70 \%$ da capacidade de campo. O lodo foi aplicado no início do experimento, sendo homogeneizado com o solo. Os frascos foram acondicionados em incubadora $\left(26^{\circ} \mathrm{C} \pm 1\right)$ durante 91 dias de incubação. A mineralização de carbono (C$\mathrm{CO}_{2}$ ) foi quantificada pela titulação do $\mathrm{NaOH}$, que reage com o $\mathrm{CO}_{2}$ (gerado pela respiração microbiana) precipitado pelo $\mathrm{BaCl}_{2}$. Foram utilizados frascos plásticos transparentes, com volume de $50 \mathrm{~mL}$, postos sobre tripés metálicos, adicionando-se $20 \mathrm{~mL}$ de $\mathrm{NaOH}$ 0,5M. Para as titulações, utilizou-se bureta digital com $\mathrm{HCl} 0,5 \mathrm{M}$, adicionando-se $2 \mathrm{~mL}$ de $\mathrm{BaCl}_{2}$ $10 \%$ e duas gotas de fenolftaleína $1 \%$. As leituras ocorreram aos $5,10,16,23,32,45,64$ e 91 dias de incubação. A cinética da mineralização do carbono foi obtida a partir da regressão exponencial: " $\mathrm{y}=\mathrm{a}$ $\left(1-\mathrm{e}^{-\mathrm{bx}}\right)$ ", ou " $\mathrm{Cm}=\mathrm{Co}\left(1-\mathrm{e}^{-\mathrm{kt}}\right)$ ", onde: $\mathrm{Cm}=$ carbono mineralizado no tempo $\mathrm{t}\left(\mathrm{em} \mathrm{mg} \mathrm{kg}^{-1}\right)$; $\mathrm{Co}=$ carbono potencialmente mineralizável (em $\left.\mathrm{mg} \mathrm{kg}^{-1}\right) ; \mathrm{k}=$ constante de velocidade da reação de mineralização (em dias); $\mathrm{t}=$ tempo em dias. Para as regressões e obtenção dos parâmetros, utilizou-se o programa SigmaPlot v.10.0.

\section{RESULTADOS E DISCUSSÃO}

A biomassa microbiana (BMS-C) reduziu proporcionalmente as doses de lodo, aumentando o quociente metabólico $\left(q \mathrm{CO}_{2}\right)$ (Figura 1a e 1b). O solo Pvd apresentou uma maior taxa de redução da BMS-C com a incubação das doses de lodo, variando entre 272,6 e 44,2mg C kg solo ${ }^{-1}$, entre os tratamentos Controle e a dose de $50 \mathrm{Mg} \mathrm{ha}^{-1}$, respectivamente; seguido do solo Pvad, variando entre 167,7 e 38,5; e do solo Pvd*, variando entre 106,8 e 53,2 entre os tratamentos Controle e a dose de $50 \mathrm{Mg}$ $\mathrm{ha}^{-1}$, respectivamente (Figura 1a). Já em relação ao $q \mathrm{CO}_{2}$, o solo Pvad apresentou os maiores valores, variando entre 1,3 e $42,6 \mathrm{mg} \mathrm{C}-\mathrm{CO}_{2} \mathrm{~g}^{-1} \mathrm{BMS}-\mathrm{C}$ hora $^{-1}$, entre os tratamentos Controle e a dose de $50 \mathrm{Mg} \mathrm{ha}^{-1}$, respectivamente, seguido do solo Pvd (0,8

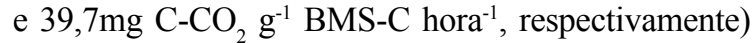
e Pvd* $\left(1,5\right.$ e 22,8mg C-CO g $^{-1}$ BMS-C hora ${ }^{-1}$,
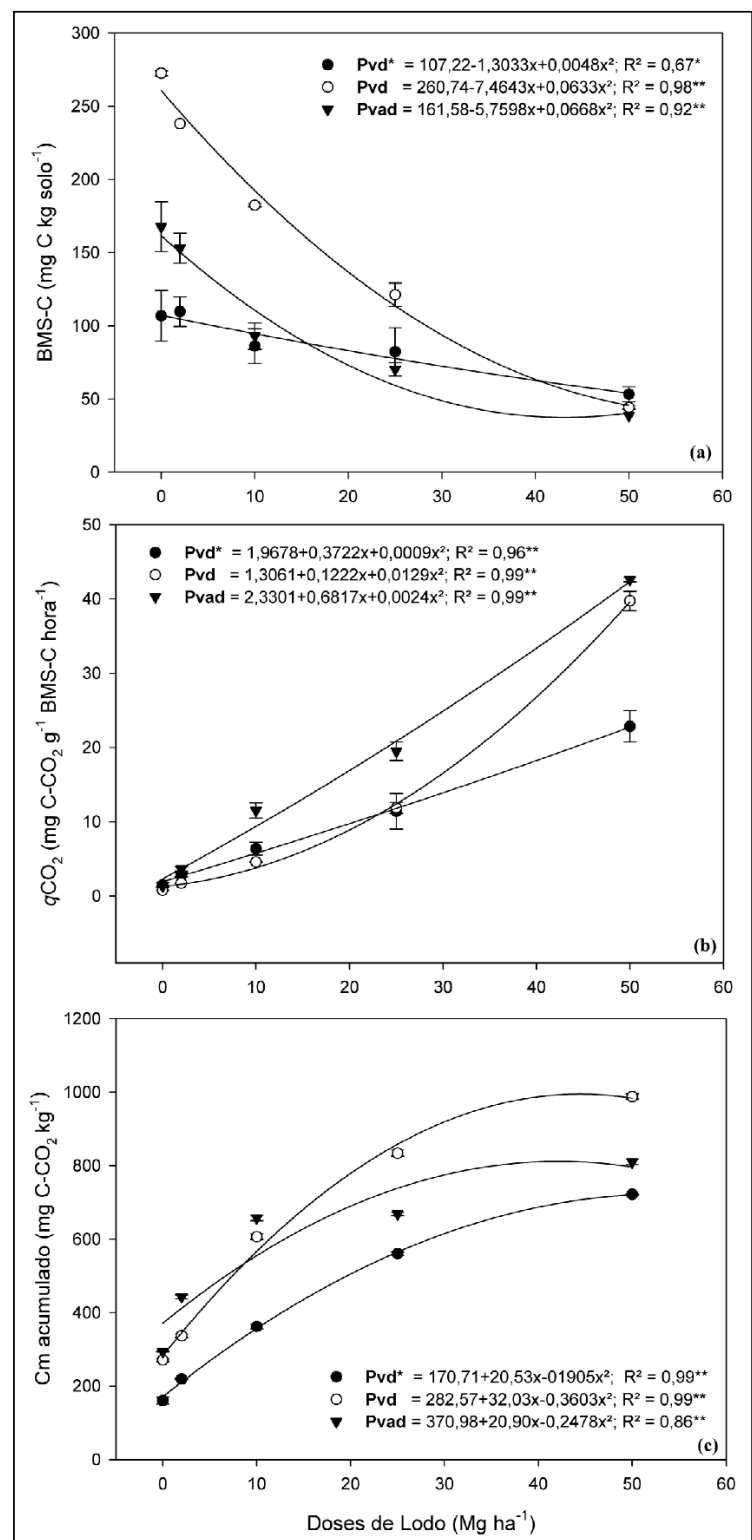

Figura 1 - Relações entre doses de lodo de ETE de aterro industrial e a biomassa microbiana do solo (BMS-C) (a); quociente metabólico do solo $\left(q \mathrm{CO}_{2}\right)$ (b); e carbono mineralizado $(\mathrm{Cm})$ acumulado (aos 91 dias de incubação) (c), nos solos $\operatorname{Pvd*}(\bullet), \operatorname{Pvd}$ $(\circ)$ e Pvad $(\boldsymbol{\nabla})$. Barras de erros são o desvio padrão. 
respectivamente). $\mathrm{O} q \mathrm{CO}_{2}$ indica que a população microbiana do solo está consumindo mais carbono oxidável para sua manutenção (ANDERSON \& DOMSCH, 2010), deste modo tornando-se menos eficiente em seus processos. A redução na BMS-C e aumento do $q \mathrm{CO}_{2}$ podem ser relacionadas ao aporte de nutrientes causado pela adição do lodo em solo, dentre estes, o sódio (TRIPATHI et al., 2006; YUAN et al., 2007), um dos elementos em maior concentração no lodo utilizado neste experimento $(2,6 \%)$. O sódio pode causar efeitos negativos diretamente, por pressão osmótica (RAIJ, 1991; McBRIDE, 1994) ou, indiretamente, em função de desordem nutricional (BISSANI et al., 2008; SPOSITO, 2008). Porém, a adição conjunta da matéria orgânica presente no lodo tende a reduzir o efeito negativo da salinidade sobre a biomassa microbiana (SILVA JUNIOR et al., 2009; MENDOZA et al., 2013). Deste modo, outros fatores podem interferir conjuntamente na BMS-C, como o aumento nas concentrações de metais pesados e a alteração no $\mathrm{pH}(\mathrm{r}=-0,76)$ e condutividade elétrica (BUENO et al., 2011), alterando as condições naturais da microbiota.

A aplicação do lodo nos solos intensificou a liberação de $\mathrm{CO}_{2}$ nos três solos, variando (no acumulado de 91 dias), no solo Pvd*, de 160,9mg $\mathrm{C}-\mathrm{CO}_{2} \mathrm{~kg} \mathrm{solo}^{-1}$ no tratamento Controle (sem aplicação de lodo) a 721,9 mg C-CO $\mathrm{kg} \mathrm{solo}^{-1}$, no tratamento com dose de lodo de $50 \mathrm{Mg} \mathrm{ha}^{-1}$; no solo Pvd, os

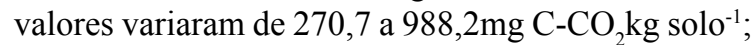
e, no solo Pvad, de 294,3 a 810,0mg C-CO kg solo $^{-1}$ (Figura 1c). Esses valores representam aumentos na mineralização de carbono de 77,$7 ; 27,6$; e $63,7 \%$, com a adição de $50 \mathrm{Mg}_{\text {lodo }}$ ha $^{-1}{ }_{\text {solo }}$, em relação ao tratamento Controle, respectivamente, para os solos Pvd*, Pvd e Pvad. Segundo BARRETO et al. (2010), a queda na inclinação das curvas de mineralização de C (Figura 1c) devem-se, possivelmente, à presença, em maior proporção, de resíduos de maior estabilidade. A intensa mineralização pode ser atribuída principalmente à baixa relação $\mathrm{C}: \mathrm{N}$ e à composição mais lábil da fração orgânica do lodo. Um segundo fator que pode influir é a remineralização, quando a microbiota remanescente utiliza como fonte de carbono, nutrientes e energia dos compostos dos micro-organismos impactados pelo estresse da modificação ambiental (MOREIRA \& SIQUEIRA, 2006; HANEY \& FRANZLUEBBERS, 2009). A mineralização do carbono foi proporcional às doses aplicadas em função do carbono orgânico presente no lodo, tendo alta correlação positiva $(r=0,85) \mathrm{com}$ esses teores. Correlações positivas ocorreram também com o $q \mathrm{CO}_{2}, \mathrm{pH}$ e condutividade elétrica $(0,82 ; 0,65$ e 0,78 , respectivamente). MARTINES et al. (2006) apontam que a maior liberação de $\mathrm{C}-\mathrm{CO}_{2}$ em solos tratados com lodo não se deve apenas à incorporação de carbono orgânico e nutrientes, mas também ao efeito corretivo e à ação inoculante do lodo. Efeitos positivos na atividade microbiana com adição de doses crescentes de lodo (da indústria de curtume) também foram encontrados por CAVALLET \& SELBACH (2008). A taxa de mineralização (k) variou no solo Pvd* de 0,5 a 2,9\% de carbono mineralizado por dia; no solo Pvd, de 0,5 a 3,8\%; e, no solo Pvad, de 0,6 a 4,1\% (Figura 2).

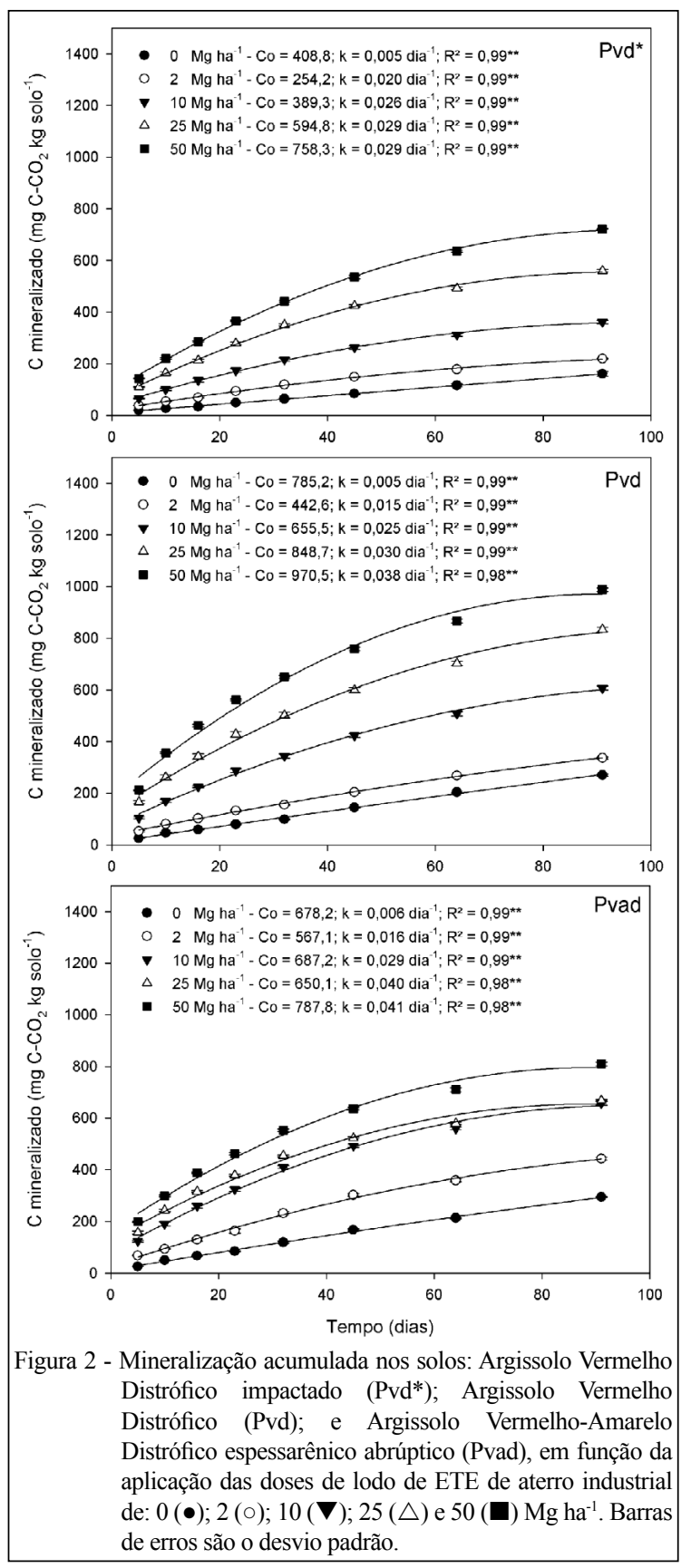


Para os três solos, não houve diferenças $(\mathrm{P}<0,005)$ entre os tratamentos Controle e a dose de $2 \mathrm{Mg} \mathrm{ha}^{-1}$, tanto para a BMS-C quanto para o $q \mathrm{CO}_{2}$. Resultados similares foram encontrados, em estudos com aplicação de resíduos orgânicos em solos, por BUENO et al. (2011) e MENDOZA et al. (2013). Efeitos positivos na atividade microbiana com adição de doses crescentes de lodo (da indústria de curtume) também foram encontrados por CAVALLET \& SELBACH (2008). De modo geral, houve aumento na atividade microbiana com as doses de lodo aplicadas. As doses não alcançaram valores limitantes para a atividade dos micro-organismos, sendo positivas até doses equivalentes a $50 \mathrm{Mg}_{\text {lodo }} \mathrm{ha}^{-1}{ }_{\text {solo }}$.

\section{CONCLUSÃO}

A atividade microbiana aumentou com a aplicação de lodo de ETE de aterro industrial, sendo a mineralização de carbono proporcional às doses adicionadas. Os resultados demonstram potencial de uso deste lodo em solo, mesmo com grande concentração de metais e sais no lodo. Entretanto, estudos de fitotoxicidade e toxicidade ambiental devem ser realizados.

\section{AGRADECIMENTOS}

Ao Conselho Nacional de Desenvolvimento Científico e Tecnológico $(\mathrm{CNPq})$, pela concessão de bolsa para o primeiro autor

\section{REFERÊNCIAS}

ASSOCIAÇÃO BRASILEIRA DE NORMAS TÉCNICAS. NBR 14283: resíduos em solos - determinação da biodegradação pelo método respirométrico. Rio de Janeiro, 1999. p10.

ANDERSON, J.P.E.; DOMSCH, K.H. A physiological method for the quantitative measurement of microbial biomass in soils. Soil Biology \& Biochemistry, Elmsford, v.10, p.215-221, 1978.

ANDERSON, T.; DOMSCH, K.H. Soil microbial biomass: the eco-physiological approach. Soil Biology \& Biochemistry, Elmsford, v.42, p.2039-2043, 2010. Disponível em: $<$ http://dx.doi. org/10.1016/j.soilbio.2010.06.026>. Acesso em: $12 \mathrm{dez}$. 2013. doi: $10.1016 /$ j.soilbio.2010.06.026.

BARRETO, P.A.B. et al. Mineralização de nitrogênio e carbono em solos sob plantações de eucalipto, em uma sequência de idades. Revista Brasileira de Ciência do Solo, Campinas, v.34, n.3, p.735-745, 2010. Disponível em: <http://dx.doi.org/10.1590/ S0100-06832010000300015>. Acesso em: 15 jan. 2014. doi: $10.1590 / \mathrm{S} 0100-06832010000300015$.

BISSANI, C.A. et al. Fertilidade dos solos e manejo da adubação de culturas. 2.ed. Porto Alegre: Gênesis, 2008. 344p.

BRASIL. Lei n.12.305, de 2 de agosto de 2010. Institui a Política Nacional de Resíduos Sólidos; altera a Lei n.9.605, de 12 de fevereiro de 1998; e dá outras providências. Diário Oficial da República Federativa do Brasil, Brasília, DF, 3 ago. 2010. Disponível em: <http://www.planalto.gov.br/ccivil_03/_ato20072010/2010/lei/112305.htm>. Acesso em: 12 out. $201 \overline{2}$.

BUENO, J.R.P. et al. Chemical and microbiological attributes of an oxisol treated with successive applications of sewage sludge. Revista Brasileira de Ciência do Solo [online], Viçosa, v.35, n.4, p.1461-1470, 2011. Disponível em: <http://dx.doi.org/10.1590/ S0100-06832011000400040>. Acesso em: 18 jan. 2014. doi: $10.1590 / \mathrm{S} 0100-06832011000400040$.

CAVALlET, L.E.; SELBACH, P.A. Populações microbianas em solo agrícola sob aplicação de lodos de curtume. Revista Brasileira de Ciência do Solo, Campinas, v.32, p.2863-2869, 2008.

COMPANHIA DE TECNOLOGIA E SANEAMENTO AMBIENTAL. Norma Técnica L 6.350: solos. Determinação da biodegradação de resíduos - Método Respirométrico de Bartha. São Paulo: CETESB, 1990. p.9.

EMBRAPA. Centro Nacional de Pesquisa de Solos. Sistema brasileiro de classificação de solos. Rio de Janeiro: Embrapa Solos, 2009. 412p.

FERREIRA, A.S. et al. Alterações de atributos químicos biológicos de solo e rendimento de milho e soja pela utilização de resíduos de curtume e carbonífero. Revista Brasileira de Ciência do Solo, Campinas, v.27, n.4, p.755-763, 2003. Disponível em: $<\mathrm{http}$ //dx.doi.org/10.1590/S0100-06832003000400020>. Acesso em: 12 set. 2013. doi: 10.1590/S0100-06832003000400020.

FERREIRA, A.S. et al. Utilização de microondas na avaliação da biomassa microbiana do solo. Revista Brasileira da Ciência do Solo, Campinas, v.23, p.991-996, 1999.

GIANELLO, C. et al. Viabilidade do uso de resíduos da agroindústria coureiro-calçadista no solo. Ciência Rural [online], Santa Maria, v.41, n.2, p.242-245, 2011. Disponível em: <http:// dx.doi.org/10.1590/S0103-84782011005000007>. Acesso em: 23 nov. 2013. doi: 10.1590/S0103-84782011005000007.

HANEY, R.L.; FRANZLUEBBERS, A.J. Soil $\mathrm{CO}_{2}$ evolution: Response from arginine additions. Applied Soil Ecology, Amsterdam, v.42, p.324-327, 2009. Disponível em: $<$ http://dx.doi. org/10.1016/j.apsoil.2009.05.004>. Acesso em: 15 set. 2013. doi:10.1016/j.apsoil.2009.05.004.

LAUSCHNER, M.H. et al. Decomposição de resíduos de agroindústria fumageira no solo. Ciência e Natura, Santa Maria, v.35 n.2, p.001-008, 2013. Disponível em: <http://dx.doi. org/10.5902/2179-460X570>. Acesso em: 15 jan. 2014. doi: $10.5902 / 2179-460 \times 570$.

MARTINES, A.M. et al. Mineralização do carbono orgânico em solos tratados com lodo de curtume. Pesquisa Agropecuária Brasileira, Brasília, v.41, n.7, p.1149-1155, 2006. Disponível em: $<$ http://dx.doi.org/10.1590/S0100-204X2006000700011>. Acesso em: 02 ago. 2013. doi: 10.1590/S0100-204X2006000700011.

McBRIDE, M.B. Environmental chemistry of soils. New York: Oxford University, 1994. Cap.vii, 406p.

MENDOZA, B. et al. Atributos biológicos de dos suelos de Quibor com aplicación de abono orgánico y soluciones salinas. Revista Mexicana de Ciencias Agrícolas, Texcoco, 
v.4, n.3, p.409-421, 2013. Disponivel em: <http://ref.scielo. org/c978fb>. Acesso em: 03 jan 2014.

MOREIRA, F.M.S.; SIQUEIRA, J.O. Microbiologia e bioquímica do solo. 2.ed. Lavras, UFLA, 2006. 729p.

PASSIANOTO, C.C. et al. Atividade e biomassa microbiana no solo com aplicação de dois diferentes lodos de curtume. Revista Brasileira Agrociência, Pelotas, v.7, n.2, p.125-130, 2001. Disponível em: <http://periodicos.ufpel.edu.br/ojs2/index.php/ CAST/article/view/384/377>. Acesso em: 10 dez. 2013.

RAIJ, B. van. Fertilidade do solo e adubação. Piracicaba: Agronômica Ceres, 1991. 343p.

SEGATTO, M.P. et al. Decomposição de resíduos industriais no solo. Ciência e Natura, Santa Maria, v.34, n.1, p.49-62, 2012. Disponível em: <http://dx.doi.org/10.5902/2179460X9354>. Acesso em: 15 nov. 2013. doi: 10.5902/2179460X9354.

SELBACH, P.A. et al. Descarte e biodegradação de lodos de curtume no solo. Revista do Couro, Estância Velha, v.17, n.79, p.51-62, 1991.

SILVA, C.A. Uso de resíduos orgânicos na agricultura. In: SANTOS, G.A. et al. (Ed.). Fundamentos da matéria orgânica do solo: ecossistemas tropicais \& subtropicais. 2.ed. rev. e atual. Porto Alegre: Metropole, 2009. p.597-624.
SILVA JUNIOR, J.M.T. et al. Efeitos de níveis de salinidade sobre a atividade microbiana de um Argissolo Amarelo incubado com diferentes adubos orgânicos. Revista Brasileira de Ciências Agrárias, Recife, v.4, n.4, p.378-382, 2009. Disponível em: $<$ http://dx.doi.org/10.5039/agraria.v4i4a1 $>$. Acesso em: 05 jan. 2014. doi: 10.5039/agraria.v4i4a1.

SPOSITO, G. The chemistry of soils. 2.ed. New York: Oxford University, 2008. 329p.

STRECK, E.V. et al. Solos do Rio Grande do Sul. 2.ed. rev. e ampl. Porto Alegre: Emater/RS, 2008. 222p.

TEDESCO M.J. et al. Análises de solos, plantas e outros materiais. 2.ed. Porto Alegre: UFRGS, Departamento de Solos, 1995. 174p.

TRIPATHI, S.S. et al. Microbial biomass and its activities in saltaffected coastal soils. Biology and Fertility of Soils, New York, v.42, p.273-277, 2006. Disponível em: <http://dx.doi.org/10.1007/ s00374-005-0037-6>. Acesso em: 10 jan. 2014. doi: 10.1007/ s00374-005-0037-6.

YUAN, B.C. et al. Microbial biomass and activity in salt affected soils under arid conditions. Applied Soil Ecology, Amsterdam, v.35, p.319-328, 2007. Disponível em: <http://dx.doi. org/10.1016/j.apsoil.2006.07.004>. Acesso em: 20 dez. 2013. doi: 10.1016/j.apsoil.2006.07.004. 